\title{
Categorization of Patients With Systemic Lupus Erythematosus Using Disease Activity, Patient- Reported Outcomes and Transcriptomic Signatures
}

\section{Robin Arcani}

AP-HM: Assistance Publique Hopitaux de Marseille

Elisabeth Jouve

AP-HM: Assistance Publique Hopitaux de Marseille

Laurent Chiche

Hopital Europeen Marseille

Noemie Jourde-Chiche ( $\nabla$ noemie.jourde@ap-hm.fr)

Aix-Marseille-University: Aix-Marseille Universite https://orcid.org/0000-0001-9315-1577

Research article

Keywords: Categorization, patients, SLE, HRQoL

Posted Date: June 8th, 2021

DOI: https://doi.org/10.21203/rs.3.rs-562655/v1

License: (9) This work is licensed under a Creative Commons Attribution 4.0 International License.

Read Full License 


\section{Abstract \\ Objective}

Patients with systemic lupus erythematosus (SLE) display symptoms that are not always related to disease activity and may distort clinical trial results. Recently, a clinical categorization based on the presence of type 1 (inflammatory manifestations) and/or type 2 (widespread pain, fatigue, depression) symptoms has been proposed in SLE. Our aim was to develop a type 2 score derived from the Short-Form health survey (SF-36) to categorize SLE patients and to compare immunological and transcriptomic profiles between groups.

\section{Methods}

Seventeen items from the SF-36 were selected to build a type 2 score for 50 SLE patients (100 visits; LUPUCE cohort) and the SLEDAI was used to define type 1 symptoms. Patients were categorized in four groups: minimal (no symptoms), type 1, type 2 and mixed (both type 1 and type 2 symptoms). Clinical, immunological and transcriptomic profiles were compared between the groups.

\section{Results}

Type 2 scores ranged from 0 to 31, with a cut-off value of 14 (75th percentile). The sample categorization was: minimal in 39\%, type 1 in 37\%, type 2 in $9 \%$ and mixed in $15 \%$. Type 2 patients were older than minimal patients and had a longer disease duration than type 1 and mixed patients. Immunological data and modular interferon signatures did not differ between the groups.

\section{Conclusion}

Patients with SLE can be categorized into four clinical groups using the SLEDAI score and our SF-36derived type 2 score. This categorization is non-redundant with immunological or transcriptomic profiles and could prove useful to stratify patients in clinical trials.

\section{Significance And Innovations}

- A score derived from selected items of the SF-36 can be used to identify SLE patients with type 2 symptoms according to the Duke University categorization.

- Using the SLEDAI and this type 2 score, SLE patients can be categorized in four clinical groups.

- This categorization is not related to immunological activity or blood transcriptome profiles (and not to the interferon signature in particular). 
- This categorization could be useful in the daily care of patients as well as in clinical trials, for upstream patient stratification or for the interpretation of results.

\section{Introduction}

Systemic lupus erythematosus (SLE) is a chronic autoimmune disease, characterized by a wide range of symptoms. Some SLE symptoms are related to systemic and local inflammation resulting from disease activity, while others can affect quiescent patients. Although survival has been improved dramatically with medications (1), patients with SLE suffer from a persistently altered health-related quality of life (HRQoL) $(2,3)$, mainly influenced by fatigue, reported by $67-90 \%$ of patients (4-7), chronic pain and depression (8). These symptoms correlate poorly with disease activity (7), do not respond to conventional immunosuppression, and are often difficult to manage. In addition, they can lead to the failure of therapeutic candidates to improve HRQoL in clinical trials (9). Recently, categorization of SLE symptoms between two non-exclusive types was proposed by Duke University (10) and tested in a cohort of 212 patients (11). Type 1 symptoms include inflammatory manifestations related to disease activity, such as nephritis, arthritis, rash, serositis and cytopenia; type 2 symptoms include widespread pain, fatigue, sleep disturbance, cognitive dysfunction and depression. This allowed the categorization of patients at the time of clinical assessment into four distinct groups (11): (i) "type 1": patients displaying only type 1 symptoms; (ii) "type 2": patients displaying only type 2 symptoms; (iii) "mixed": patients displaying both type 1 and type 2 symptoms; (iv) "minimal": patients with no symptoms. This classification was built upon the SLEDAl score (type 1 symptoms), which is widely available in SLE studies, and on American College of Rheumatology (ACR) fibromyalgia criteria (12) (type 2 symptoms) (11), which are less commonly used in SLE and are rarely available retrospectively.

Our aim was to determine whether selected items from the SF-36 questionnaire could allow the identification of type 2 symptoms and the retrospective categorization of patients from different SLE cohorts. We then aimed to evaluate whether these categories were associated with distinct immunological and transcriptomic profiles. In particular, we compared the interferon (IFN) modular scores of samples from these four groups through a modular framework of analysis described previously (13).

\section{Patients And Methods}

\section{Patients and Ethics}

This study involved data from patients included in the LUPUCE study (NCT00920114) and the PSY-LUP study (NCT03913754). All patients gave their written informed consent before any study-related procedure. The study was conducted according to the Declaration of Helsinki.

LUPUCE was a transcriptomic study (13-15) comprising 62 consecutive SLE patients, of whom 50 had completed the self-administered SF-36 questionnaire on at least at one visit and were included in the present study. Clinical, biological and transcriptomic data were recorded at each visit. The study was 
approved in France by the Comité de Protection des Personnes Sud Méditerranée 1 (IDRCB 2009-A0025750) and in the USA by the Institutional Review Boards (IRBs) of the Baylor Institute of Immunology Research (IRB 011-173) and the Benaroya Research Institute (IRB 12085).

PSY-LUP (NCT03913754) is a study evaluating the psycho-social consequences of SLE. One-hundred SLE patients responded to several quality of life (QoL) questionnaires including the SF-36. The study was approved by the Comité de Protection des Personnes lle de France I (IDRCB A02747-48).

\section{Assessment of type 1 and type 2 symptoms}

Patients were considered to have type 1 symptoms if they had a SLEDAI $\geq 6$, a clinical SLEDAI $\geq 4$, or active lupus nephritis documented by kidney biopsy (11).

The presence of type 2 symptoms was defined using a score built from the responses to specific items of the SF-36 questionnaire (16) (detailed in Table 1). Three physicians experienced in SLE (RA, LC and NJC) manually selected 17 items/questions they considered the most relevant to express widespread pain, fatigue and depression. Responses to these items were weighted and the global type 2 score ranged from 0 to 42. The 75th percentile of this score in the LUPUCE cohort was chosen as the threshold to consider that a patient displayed significant type 2 symptoms. 
Table 1

Detailed type 2 score, built from the responses to 17 items of the SF-36.

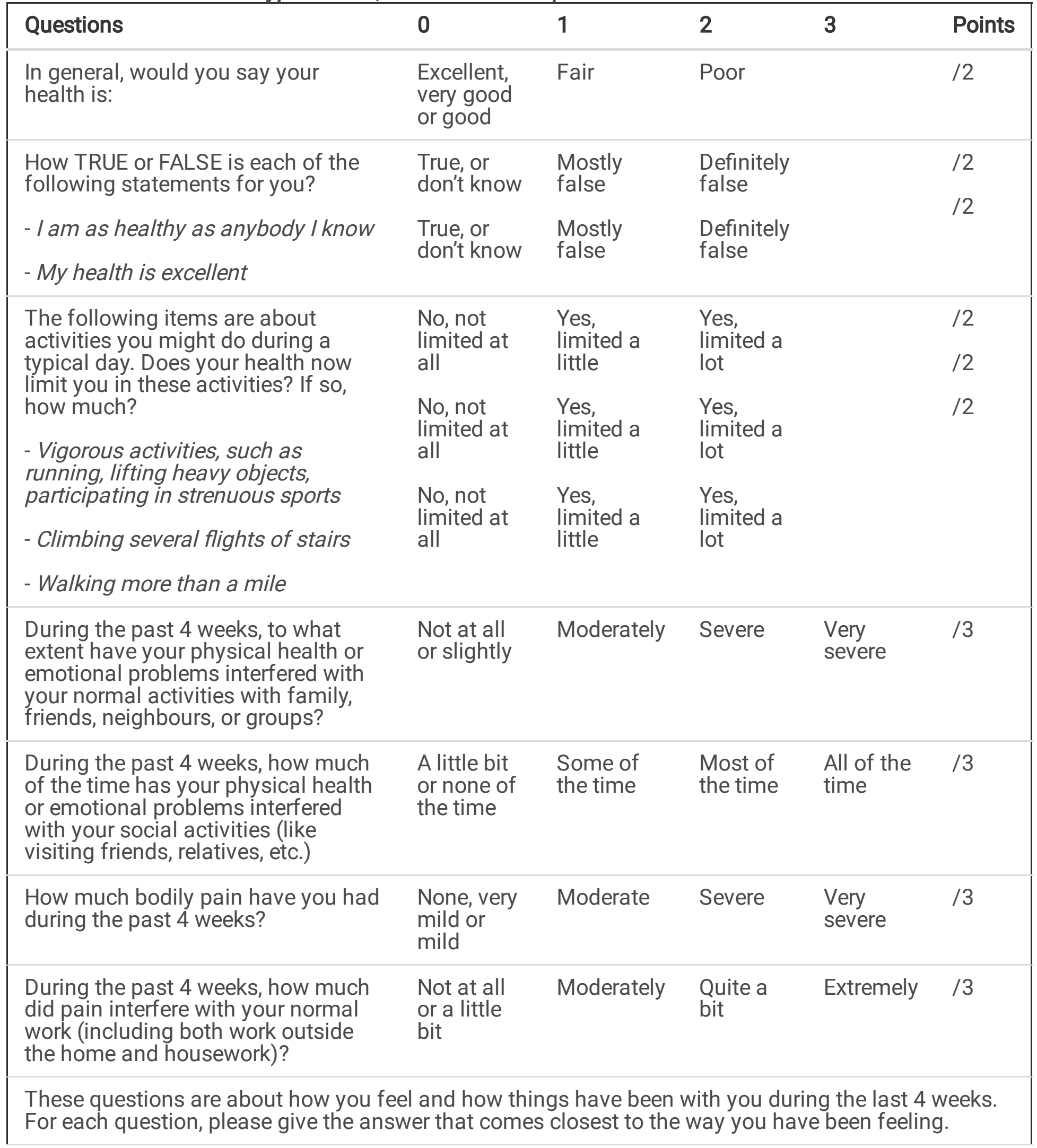




\begin{tabular}{|c|c|c|c|c|c|}
\hline Questions & 0 & 1 & 2 & 3 & Points \\
\hline $\begin{array}{l}\text { How much of the time during the } \\
\text { past } 4 \text { weeks: } \\
\text { - Did you feel full of pep? } \\
\text { - Have you felt so down in the } \\
\text { dumps that nothing could cheer you } \\
\text { up? } \\
\text { - Did you have a lot of energy? } \\
\text { - Have you felt downhearted and } \\
\text { blue? } \\
\text { - Did you feel worn out? } \\
\text { - Have you been a happy person? } \\
\text { - Did you feel tired? }\end{array}$ & $\begin{array}{l}\text { Some, a } \\
\text { little bit or } \\
\text { none of } \\
\text { the time } \\
\text { All, most, a } \\
\text { good bit or } \\
\text { some of } \\
\text { the time } \\
\text { Some, a } \\
\text { little bit or } \\
\text { none of } \\
\text { the time } \\
\text { Some, a } \\
\text { little bit or } \\
\text { none of } \\
\text { the time } \\
\text { All, most, a } \\
\text { good bit or } \\
\text { some of } \\
\text { the time } \\
\text { Some, a } \\
\text { little bit or } \\
\text { none of } \\
\text { the time }\end{array}$ & $\begin{array}{l}\text { A little bit } \\
\text { of the time } \\
\text { A good bit } \\
\text { of the time } \\
\text { A good bit } \\
\text { of the time } \\
\text { A little bit } \\
\text { of the time } \\
\text { A good bit } \\
\text { of the rime }\end{array}$ & $\begin{array}{l}\text { None of } \\
\text { the time } \\
\text { Most of } \\
\text { the time } \\
\text { Most of } \\
\text { the time } \\
\text { None of } \\
\text { the time } \\
\text { Most of } \\
\text { the time }\end{array}$ & $\begin{array}{l}\text { All of the } \\
\text { time } \\
\text { All of the } \\
\text { time }\end{array}$ & $\begin{array}{l}/ 3 \\
/ 3 \\
12\end{array}$ \\
\hline Total Score & & & & & $/ 42$ \\
\hline
\end{tabular}

\section{Immunological activity}

Immunological activity was evaluated by the levels of the complement fractions C3 and/or C4 and of anti-dsDNA antibodies.

\section{Blood modular transcriptional repertoire analyses}

Whole-blood transcriptomic data collected in the LUPUCE study were investigated (13). RNA was processed as described elsewhere using Illumina beadchips (13). Data are deposited in the NCBI Gene Expression Omnibus (GEO, http://www.ncbi.nlm.nih.gov/geo, GEO Series accession number GSE49454). Analyses were performed using the second generation of a modular framework as described previously $(13,17)$. The level of regulation of each module was calculated as the percentage difference: \% upregulated probes - \% downregulated probes. A module was considered activated (up-regulated) or inhibited (down-regulated) if its value was, respectively, $\geq 20 \%$ or $\leq-20 \%$. A modular IFN score was 
calculated depending on the activation of three IFN modules (M1.2, M3.4 and M5.12). The IFN signature was absent, mild, moderate or strong if $0,1,2$ or 3 IFN modules were active ( $\geq 20 \%)$ respectively (13).

\section{Statistical analysis}

Quantitative variables are described as median and interquartile range (IQR), or mean \pm standard deviation (SD); categorical variables are described as numbers and percentages.

The four clinical groups were compared for patient characteristics, immunological activity and level of regulation of IFN modules (modules M1.2, M3.4 and M5.12) using the Kruskal Wallis test and Tukey's test for pairwise comparisons. Categorical patient characteristics, frequency of IFN signature and active modules were compared between the four clinical groups using the $\mathrm{Chi}^{2}$ test or Fisher's exact test. Exploratory analysis compared levels of regulation of all modules between the four clinical groups using the Kruskal-Wallis test with false discovery rate (FDR) correction for multiplicity. Similarly, the presence of modules was compared using Fisher's exact test with FDR correction. Statistical tests were two-tailed and significance was set at $p<0.05$. All statistical analyses were conducted using IBM SPSS Statistics 20.

\section{Results}

\section{Type 2 score and patient distribution between clinical groups}

All visits $(n=100)$ at which patients from the LUPUCE cohort answered a SF-36 questionnaire were considered to calculate the type 2 score (Table 1). The type 2 score ranged from 0 to 31 , with a median of 6 and interquartile range [IQR] of 3-14. The threshold to define type 2 symptoms was $>14$. Median SLEDAI was 4 [IQR: 0-22] and patients exhibited type 1 symptoms at 52 visits. Patients from the LUPUCE cohort were categorized into four clinical groups: minimal in 39\%, type 1 in 37\%, type 2 in $9 \%$ and mixed in $15 \%$ (Table 2).

Table 2

Distribution (\%) of SLE patients from different cohorts in the four clinical groups.

\begin{tabular}{|llll|}
\hline Clinical groups & $\begin{array}{l}\text { LUPUCE cohort } \\
(\mathbf{N}=\mathbf{1 0 0})\end{array}$ & $\begin{array}{l}\text { Duke University cohort } \\
(\mathbf{N}=\mathbf{2 1 2})\end{array}$ & $\begin{array}{l}\text { PSY-LUP cohort } \\
(\mathbf{N}=\mathbf{1 0 0})\end{array}$ \\
\hline Minimal & 39 & 49 & ND \\
\hline Type 1 & 37 & 30 & ND \\
\cline { 1 - 3 } 2 & 9 & 8 & 23 \\
\hline Mixed & 15 & 13 & \\
\hline ND: not determined (the SLEDAl was not recorded in the PSY-LUP cohort)
\end{tabular}


The type 2 score was calculated in an independent cohort of 100 SLE patients (PSY-LUP cohort). This showed a similar pattern of values to the LUPUCE cohort (Fig. 1): the score ranged from 0 to 33, with a median of 8.3 , and $23 \%$ of patients were above the threshold of 14 . The distribution of patients from the LUPUCE cohort was compared with that from Duke University (11), in which type 2 symptoms were defined by ACR fibromyalgia criteria. A similar distribution was found in the two cohorts (Table 2).

The characteristics of patients from the LUPUCE cohort at their first visit ( $n=50$ unique patients) and the characteristics of patient-by-visit by clinical groups are summarized in Table 3. The median time between each visit was 124 days [IQR: 20-130]. Patients in the "type 2" group were older (52 vs. 31 years, $p=$ $0.016)$ and more often menopausal $(66.7 \%$ vs. $17.9 \%, p=0.031)$ than patients in the "minimal" group, and they had a longer disease duration than patients in the "type 1" and "mixed" groups (10 vs. 5 years and 10 vs. 2 years, $p=0.011$, respectively). 
Table 3

Characteristics of patients at their first visit (unique patient, $N=50)$ and of patient-by-visit ( $N=100$ visits) in the four clinical groups.

\begin{tabular}{|c|c|c|c|c|c|c|c|}
\hline & $\begin{array}{l}\begin{array}{l}\text { Total } \\
\text { patients }\end{array} \\
n=50\end{array}$ & $\begin{array}{l}\text { Minimal } \\
n=39\end{array}$ & $\begin{array}{l}\text { Type-1 } \\
n=37\end{array}$ & $\begin{array}{l}\text { Type- } \\
2 \\
n=9\end{array}$ & $\begin{array}{l}\text { Mixed } \\
n=15\end{array}$ & $\begin{array}{l}\text { Total } \\
\text { visits } \\
n= \\
100\end{array}$ & $p$ value \\
\hline Number of visits & $2[1-5]$ & $2[1-4]$ & $\begin{array}{l}2[1- \\
4]\end{array}$ & $\begin{array}{l}1[1- \\
3]\end{array}$ & $\begin{array}{l}1[1- \\
3]\end{array}$ & $\begin{array}{l}1.5 \\
{[1-5]}\end{array}$ & \\
\hline Sex, female & $\begin{array}{l}43 \\
(86)\end{array}$ & $\begin{array}{l}31 \\
(79.5)\end{array}$ & $\begin{array}{l}33 \\
(89.2)\end{array}$ & $\begin{array}{l}9 \\
(100)\end{array}$ & $\begin{array}{l}10 \\
(66.7)\end{array}$ & $\begin{array}{l}83 \\
(83)\end{array}$ & 0.119 \\
\hline Age (years) & $\begin{array}{l}38 \\
{[18-70]}\end{array}$ & $\begin{array}{l}31 \\
{[18-54]}\end{array}$ & $\begin{array}{l}36 \\
{[20-} \\
67]\end{array}$ & $\begin{array}{l}52 \\
{[39-} \\
70]\end{array}$ & $\begin{array}{l}44 \\
{[25-} \\
56]\end{array}$ & $\begin{array}{l}37 \\
{[18-} \\
70]\end{array}$ & $0.016^{a}$ \\
\hline $\begin{array}{l}\text { Ethnicity } \\
\text { Caucasian }\end{array}$ & $\begin{array}{l}44 \\
(88)\end{array}$ & $\begin{array}{l}35 \\
(89.7)\end{array}$ & $\begin{array}{l}30 \\
(81.1)\end{array}$ & $\begin{array}{l}9 \\
(100)\end{array}$ & $\begin{array}{l}15 \\
(100)\end{array}$ & $\begin{array}{l}89 \\
(89)\end{array}$ & 0.636 \\
\hline Black & $3(6)$ & $2(5.1)$ & $2(5.4)$ & $0(0)$ & $0(0)$ & $4(4)$ & \\
\hline Asian & $3(6)$ & $2(5.1)$ & $\begin{array}{l}5 \\
(13.5)\end{array}$ & $0(0)$ & $0(0)$ & $7(7)$ & \\
\hline Hypertension & $21(42)$ & $\begin{array}{l}17 \\
(43.6)\end{array}$ & $\begin{array}{l}17 \\
(45.9)\end{array}$ & $\begin{array}{l}5 \\
(55.6)\end{array}$ & $9(60)$ & $\begin{array}{l}48 \\
(48)\end{array}$ & 0.723 \\
\hline Smoking & $16(32)$ & $\begin{array}{l}11 \\
(28.2)\end{array}$ & $\begin{array}{l}17 \\
(45.9)\end{array}$ & $\begin{array}{l}1 \\
(11.1)\end{array}$ & $\begin{array}{l}7 \\
(46.7)\end{array}$ & $\begin{array}{l}36 \\
(36)\end{array}$ & 0.126 \\
\hline Menopause & $16(32)$ & $7(17.9)$ & $\begin{array}{l}13 \\
(35.1)\end{array}$ & $\begin{array}{l}6 \\
(66.7)\end{array}$ & $\begin{array}{l}4 \\
(26.7)\end{array}$ & $\begin{array}{l}30 \\
(30)\end{array}$ & $0.031^{a}$ \\
\hline Antiphospholipid Sd & $5(10)$ & $7(17.9)$ & $\begin{array}{l}5 \\
(13.5)\end{array}$ & $0(0)$ & $\begin{array}{l}2 \\
(13.3)\end{array}$ & $\begin{array}{l}14 \\
(14)\end{array}$ & 0.670 \\
\hline $\begin{array}{l}\text { Disease duration } \\
\text { (years) }\end{array}$ & $7[0-40]$ & $\begin{array}{l}7[1- \\
24]\end{array}$ & $\begin{array}{l}5[0- \\
30]\end{array}$ & $\begin{array}{l}10[3- \\
40]\end{array}$ & $\begin{array}{l}2[0- \\
22]\end{array}$ & $\begin{array}{l}7[0- \\
40]\end{array}$ & $0.011^{b, c}$ \\
\hline
\end{tabular}

Values shown are median [IQR], or $\mathrm{n}(\%)$.

SLE: systemic lupus erythematosus; $\mathrm{Hb}$ : haemoglobin.

p value: comparison between SLE subtypes (Kruskal Wallis test), significant pairwise comparisons (Tukey test): ${ }^{\text {a: }}$ Minimal < Type 2; : Type $1<$ Type 2; : Mixed < Type 2; : Minimal < Type 1; e: Minimal<

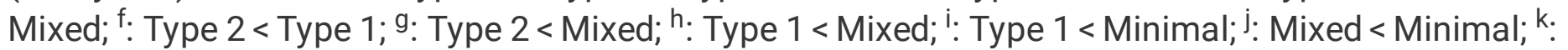
Mixed < Type 1 ; : Type $2<$ Minimal 


\begin{tabular}{|c|c|c|c|c|c|c|c|}
\hline & $\begin{array}{l}\text { Total } \\
\text { patients } \\
n=50\end{array}$ & $\begin{array}{l}\text { Minimal } \\
n=39\end{array}$ & $\begin{array}{l}\text { Type-1 } \\
n=37\end{array}$ & $\begin{array}{l}\text { Type- } \\
2 \\
n=9\end{array}$ & $\begin{array}{l}\text { Mixed } \\
n=15\end{array}$ & $\begin{array}{l}\text { Total } \\
\text { visits } \\
n= \\
100\end{array}$ & $\mathrm{p}$ value \\
\hline SLEDAI & $4[0-22]$ & $2[0-4]$ & $\begin{array}{l}10[4- \\
22]\end{array}$ & $\begin{array}{l}2[0- \\
4]\end{array}$ & $\begin{array}{l}14[4- \\
22]\end{array}$ & $\begin{array}{l}4[0- \\
22]\end{array}$ & $\begin{array}{l}< \\
0.001^{\mathrm{d}, \mathrm{e}, \mathrm{f}, \mathrm{g}, \mathrm{h}}\end{array}$ \\
\hline Flaring SLE & $27(54)$ & $5(12.8)$ & $\begin{array}{l}32 \\
(86.5)\end{array}$ & $\begin{array}{l}1 \\
(11.1)\end{array}$ & $\begin{array}{l}15 \\
(100)\end{array}$ & $\begin{array}{l}55 \\
(55)\end{array}$ & $<0.001^{b, c, i, j}$ \\
\hline Cutaneous flare & $13(26)$ & $2(5.1)$ & $\begin{array}{l}14 \\
(37.8)\end{array}$ & $\begin{array}{l}1 \\
(11.1)\end{array}$ & $6(40)$ & $\begin{array}{l}23 \\
(23)\end{array}$ & $0.001^{\mathrm{d}, \mathrm{e}}$ \\
\hline Articular flare & $10(20)$ & $0(0)$ & $\begin{array}{l}11 \\
(29.7)\end{array}$ & $0(0)$ & $\begin{array}{l}5 \\
(33.3)\end{array}$ & $\begin{array}{l}16 \\
(16)\end{array}$ & $0.001^{\mathrm{d}, \mathrm{e}}$ \\
\hline Haematological flare & $4(8)$ & $1(2.6)$ & $\begin{array}{l}5 \\
(13.5)\end{array}$ & $0(0)$ & $\begin{array}{l}2 \\
(13.3)\end{array}$ & $8(8)$ & 0.190 \\
\hline Renal flare & $17(34)$ & $0(0)$ & $\begin{array}{l}19 \\
(51.4)\end{array}$ & $0(0)$ & $\begin{array}{l}12 \\
(80)\end{array}$ & $\begin{array}{l}31 \\
(31)\end{array}$ & $\begin{array}{l}< \\
0.001^{\mathrm{d}, \mathrm{e}, \mathrm{f}, \mathrm{g}}\end{array}$ \\
\hline \multirow{2}{*}{$\begin{array}{l}\text { Serum creatinine } \\
(\mu \mathrm{mol} / \mathrm{l})\end{array}$} & 70 & 71 & 61 & 73 & 102 & 70 & \multirow[t]{2}{*}{0.087} \\
\hline & {$[42-514]$} & $\begin{array}{l}{[42-} \\
150]\end{array}$ & $\begin{array}{l}{[48-} \\
100]\end{array}$ & $\begin{array}{l}{[58-} \\
122]\end{array}$ & $\begin{array}{l}{[51-} \\
514]\end{array}$ & $\begin{array}{l}{[42-} \\
514]\end{array}$ & \\
\hline CKD & $10(20)$ & $6(15.4)$ & $\begin{array}{l}6 \\
(16.2)\end{array}$ & $\begin{array}{l}3 \\
(33.3)\end{array}$ & $3(20)$ & $\begin{array}{l}18 \\
(18)\end{array}$ & 0.596 \\
\hline AKI & $5(10)$ & $0(0)$ & $1(2.7)$ & $0(0)$ & $\begin{array}{l}5 \\
(33.3)\end{array}$ & $6(6)$ & $<0.001^{\mathrm{eh}}$ \\
\hline Nephrotic Sd & $10(20)$ & $0(0)$ & $\begin{array}{l}7 \\
(18.9)\end{array}$ & $0(0)$ & $6(40)$ & $\begin{array}{l}13 \\
(13)\end{array}$ & $<0.001^{\mathrm{d}, \mathrm{e}}$ \\
\hline Anti-dsDNA + & $34(68)$ & $\begin{array}{l}23 \\
(60.5)\end{array}$ & $\begin{array}{l}22 \\
(59.5)\end{array}$ & $\begin{array}{l}8 \\
(88.9)\end{array}$ & $\begin{array}{l}10 \\
(71.4)\end{array}$ & $\begin{array}{l}63 \\
(64.3)\end{array}$ & 0.377 \\
\hline \multirow[t]{2}{*}{ Anti-dsDNA level } & 43 & 24 & 34 & 60 & 47 & 39 & \multirow[t]{2}{*}{0.446} \\
\hline & [0-578] & [0-465] & $\begin{array}{l}{[1-} \\
578]\end{array}$ & $\begin{array}{l}{[2-} \\
110]\end{array}$ & $\begin{array}{l}{[1-} \\
400]\end{array}$ & $\begin{array}{l}{[0-} \\
578]\end{array}$ & \\
\hline
\end{tabular}

Values shown are median [IQR], or $\mathrm{n}(\%)$.

SLE: systemic lupus erythematosus; $\mathrm{Hb}$ : haemoglobin.

p value: comparison between SLE subtypes (Kruskal Wallis test), significant pairwise comparisons (Tukey test): ${ }^{\text {a: }}$ Minimal < Type 2; : Type $1<$ Type 2; : Mixed < Type 2; : Minimal < Type 1; e: Minimal<

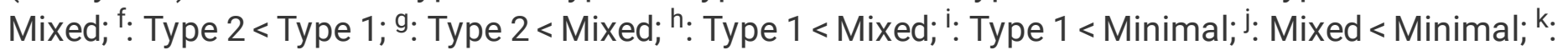
Mixed $<$ Type $1 ;$ : : Type $2<$ Minimal 


\begin{tabular}{|c|c|c|c|c|c|c|c|}
\hline & $\begin{array}{l}\begin{array}{l}\text { Total } \\
\text { patients }\end{array} \\
n=50\end{array}$ & $\begin{array}{l}\text { Minimal } \\
n=39\end{array}$ & $\begin{array}{l}\text { Type-1 } \\
n=37\end{array}$ & $\begin{array}{l}\text { Type- } \\
2 \\
n=9\end{array}$ & $\begin{array}{l}\text { Mixed } \\
n=15\end{array}$ & $\begin{array}{l}\text { Total } \\
\text { visits } \\
n= \\
100\end{array}$ & $\mathrm{p}$ value \\
\hline Anti-RNP + & $12(24)$ & $5(12.8)$ & $\begin{array}{l}6 \\
(16.2)\end{array}$ & $\begin{array}{l}2 \\
(22.2)\end{array}$ & $\begin{array}{l}4 \\
(26.7)\end{array}$ & $\begin{array}{l}17 \\
(17)\end{array}$ & 0.568 \\
\hline Anti-Sm + & $7(14)$ & $5(12.8)$ & $2(5.4)$ & $\begin{array}{l}2 \\
(22.2)\end{array}$ & $3(20)$ & $\begin{array}{l}12 \\
(12)\end{array}$ & 0.233 \\
\hline Anti-SSA + & $\begin{array}{l}18 \\
(36)\end{array}$ & $\begin{array}{l}13 \\
(33.3)\end{array}$ & $\begin{array}{l}18 \\
(48.6)\end{array}$ & $\begin{array}{l}2 \\
(22.2)\end{array}$ & $\begin{array}{l}7 \\
(46.7)\end{array}$ & $\begin{array}{l}40 \\
(40)\end{array}$ & 0.368 \\
\hline Anti-SSB + & $4(8)$ & $1(2.6)$ & $\begin{array}{l}4 \\
(10.8)\end{array}$ & $0(0)$ & $1(6.7)$ & $6(6)$ & 0.405 \\
\hline Low C3 & $\begin{array}{l}21 \\
(42)\end{array}$ & $\begin{array}{l}15 \\
(38.5)\end{array}$ & $\begin{array}{l}21 \\
(56.8)\end{array}$ & $\begin{array}{l}3 \\
(33.3)\end{array}$ & $\begin{array}{l}4 \\
(26.7)\end{array}$ & $\begin{array}{l}43 \\
(43)\end{array}$ & 0.171 \\
\hline Low C4 & $\begin{array}{l}14 \\
(28)\end{array}$ & $\begin{array}{l}12 \\
(30.8)\end{array}$ & $\begin{array}{l}14 \\
(37.8)\end{array}$ & $\begin{array}{l}3 \\
(33.3)\end{array}$ & $\begin{array}{l}5 \\
(33.3)\end{array}$ & $\begin{array}{l}34 \\
(34)\end{array}$ & 0.954 \\
\hline Low C3 or C4 & $\begin{array}{l}23 \\
(46)\end{array}$ & $\begin{array}{l}15 \\
(38.5)\end{array}$ & $\begin{array}{l}23 \\
(62.2)\end{array}$ & $\begin{array}{l}3 \\
(33.3)\end{array}$ & $6(40)$ & $\begin{array}{l}47 \\
(47)\end{array}$ & 0.148 \\
\hline $\mathrm{Hb}<12 \mathrm{~g} / \mathrm{dL}$ & $\begin{array}{l}18 \\
(36)\end{array}$ & $9(23.7)$ & $\begin{array}{l}8 \\
(21.6)\end{array}$ & $\begin{array}{l}3 \\
(33.3)\end{array}$ & $9(60)$ & $\begin{array}{l}29 \\
(29.3)\end{array}$ & $0.042^{b, e, h}$ \\
\hline Hydroxychloroquine & $\begin{array}{l}28 \\
(56)\end{array}$ & $\begin{array}{l}21 \\
(53.8)\end{array}$ & $\begin{array}{l}24 \\
(64.9)\end{array}$ & $\begin{array}{l}6 \\
(66.7)\end{array}$ & $6(40)$ & $\begin{array}{l}57 \\
(57)\end{array}$ & 0.357 \\
\hline Corticosteroids & $\begin{array}{l}43 \\
(86)\end{array}$ & $\begin{array}{l}34 \\
(87.2)\end{array}$ & $\begin{array}{l}32 \\
(86.5)\end{array}$ & $\begin{array}{l}9 \\
(100)\end{array}$ & $\begin{array}{l}14 \\
(93.3)\end{array}$ & $\begin{array}{l}89 \\
(89)\end{array}$ & 0.835 \\
\hline $\begin{array}{l}\text { Corticosteroids } \\
\text { (mg/day) }\end{array}$ & $\begin{array}{l}8 \\
{[0-90]}\end{array}$ & $\begin{array}{l}5[0- \\
20]\end{array}$ & $\begin{array}{l}15[0- \\
60]\end{array}$ & $\begin{array}{l}8[5- \\
8]\end{array}$ & $\begin{array}{l}20[0- \\
90]\end{array}$ & $\begin{array}{l}8[0- \\
90]\end{array}$ & $0.003^{e, g}$ \\
\hline
\end{tabular}

Values shown are median [IQR], or n (\%).

SLE: systemic lupus erythematosus; $\mathrm{Hb}$ : haemoglobin.

p value: comparison between SLE subtypes (Kruskal Wallis test), significant pairwise comparisons

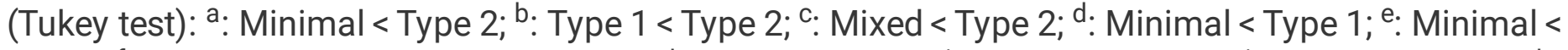

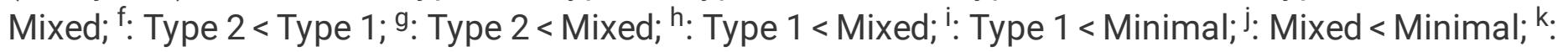
Mixed < Type 1 ; : Type $2<$ Minimal 


\begin{tabular}{|c|c|c|c|c|c|c|c|}
\hline & $\begin{array}{l}\text { Total } \\
\text { patients } \\
n=50\end{array}$ & $\begin{array}{l}\text { Minimal } \\
n=39\end{array}$ & $\begin{array}{l}\text { Type-1 } \\
n=37\end{array}$ & $\begin{array}{l}\text { Type- } \\
2 \\
n=9\end{array}$ & $\begin{array}{l}\text { Mixed } \\
n=15\end{array}$ & $\begin{array}{l}\text { Total } \\
\text { visits } \\
n= \\
100\end{array}$ & $p$ value \\
\hline Cyclophosphamide & $4(8)$ & $0(0)$ & $\begin{array}{l}4 \\
(10.8)\end{array}$ & $0(0)$ & $1(6.7)$ & $5(5)$ & 0.171 \\
\hline Azathioprine & $14(28)$ & $\begin{array}{l}14 \\
(35.9)\end{array}$ & $\begin{array}{l}11 \\
(29.7)\end{array}$ & $\begin{array}{l}5 \\
(55.6)\end{array}$ & $6(40)$ & $\begin{array}{l}36 \\
(36)\end{array}$ & 0.526 \\
\hline $\begin{array}{l}\text { Mycophenolate } \\
\text { Mofetil }\end{array}$ & $7(14)$ & $7(17.9)$ & $\begin{array}{l}4 \\
(10.8)\end{array}$ & $0(0)$ & $3(20)$ & $\begin{array}{l}14 \\
(14)\end{array}$ & 0.515 \\
\hline \multirow[t]{2}{*}{ Physical functioning } & 80 & 95 & 90 & 60 & 45 & 85 & $<0.001^{\mathrm{f}, \mathrm{j}, \mathrm{k}, \mathrm{l}}$ \\
\hline & [5-100] & $\begin{array}{l}{[60-} \\
100]\end{array}$ & $\begin{array}{l}{[28-} \\
100]\end{array}$ & $\begin{array}{l}{[10-} \\
90]\end{array}$ & [5-85] & $\begin{array}{l}{[5-} \\
100]\end{array}$ & \\
\hline \multirow[t]{2}{*}{ Social functioning } & 63 & 75 & 63 & 38 & 50 & 63 & $<0.001^{\mathrm{fj}, \mathrm{j}, \mathrm{l}, \mathrm{l}}$ \\
\hline & [0-100] & $\begin{array}{l}{[50-} \\
100]\end{array}$ & $\begin{array}{l}{[25-} \\
100]\end{array}$ & {$[0-75]$} & $\begin{array}{l}{[13-} \\
88]\end{array}$ & $\begin{array}{l}{[0-} \\
100]\end{array}$ & \\
\hline \multirow[t]{2}{*}{ Role physical } & 75 & 100 & 100 & 25 & 0 & 100 & $<0.001^{\mathrm{fj}, \mathrm{k}, \mathrm{l}, \mathrm{l}}$ \\
\hline & [0-100] & [0-100] & $\begin{array}{l}{[0-} \\
100]\end{array}$ & $\begin{array}{l}{[0-} \\
100]\end{array}$ & $\begin{array}{l}{[0-} \\
100]\end{array}$ & $\begin{array}{l}{[0-} \\
100]\end{array}$ & \\
\hline \multirow[t]{2}{*}{ Role emotional } & 100 & 100 & 100 & 0 & 33 & 100 & $0.003^{\mathrm{fj}, \mathrm{l}}$ \\
\hline & {$[0-100]$} & [0-100] & $\begin{array}{l}{[0-} \\
100]\end{array}$ & $\begin{array}{l}{[0-} \\
100]\end{array}$ & $\begin{array}{l}{[0-} \\
100]\end{array}$ & $\begin{array}{l}{[0-} \\
100]\end{array}$ & \\
\hline \multirow[t]{2}{*}{ Mental health } & 60 & 64 & 64 & 40 & 44 & 60 & $0.014^{\prime}$ \\
\hline & [24-96] & [28-92] & $\begin{array}{l}{[28-} \\
88]\end{array}$ & $\begin{array}{l}{[28-} \\
80]\end{array}$ & $\begin{array}{l}{[24-} \\
96]\end{array}$ & $\begin{array}{l}{[24-} \\
96]\end{array}$ & \\
\hline \multirow[t]{2}{*}{ Vitality } & 45 & 55 & 50 & 30 & 25 & 48 & $<0.001^{f, j, k, l}$ \\
\hline & [0-90] & $\begin{array}{l}{[15-} \\
100]\end{array}$ & $\begin{array}{l}{[30-} \\
100]\end{array}$ & $\begin{array}{l}{[10-} \\
45]\end{array}$ & {$[0-55]$} & $\begin{array}{l}{[0-} \\
100]\end{array}$ & \\
\hline
\end{tabular}

Values shown are median [IQR], or $\mathrm{n}(\%)$.

SLE: systemic lupus erythematosus; $\mathrm{Hb}$ : haemoglobin.

p value: comparison between SLE subtypes (Kruskal Wallis test), significant pairwise comparisons

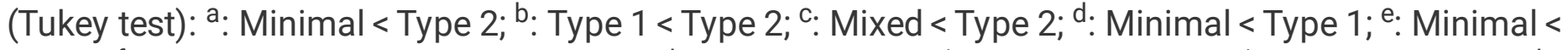

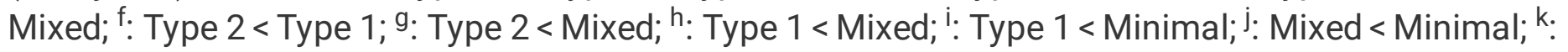
Mixed < Type 1; : Type $2<$ Minimal 


\begin{tabular}{|c|c|c|c|c|c|c|c|}
\hline & $\begin{array}{l}\text { Total } \\
\text { patients } \\
n=50\end{array}$ & $\begin{array}{l}\text { Minimal } \\
n=39\end{array}$ & $\begin{array}{l}\text { Type-1 } \\
n=37\end{array}$ & $\begin{array}{l}\text { Type- } \\
2 \\
n=9\end{array}$ & $\begin{array}{l}\text { Mixed } \\
n=15\end{array}$ & $\begin{array}{l}\text { Total } \\
\text { visits } \\
n= \\
100\end{array}$ & $\mathrm{p}$ value \\
\hline \multirow[t]{2}{*}{ Bodily pain } & 61 & 84 & 62 & 32 & 41 & 62 & $<0.001^{\mathrm{f}, \mathrm{j}, \mathrm{k}, \mathrm{l}}$ \\
\hline & {$[0-100]$} & $\begin{array}{l}{[22-} \\
100]\end{array}$ & $\begin{array}{l}{[22-} \\
100]\end{array}$ & {$[0-74]$} & {$[0-52]$} & $\begin{array}{l}{[0-} \\
100]\end{array}$ & \\
\hline \multirow[t]{2}{*}{ General health } & 54 & 57 & 57 & 25 & 25 & 54 & $<0.001^{\mathrm{fj}, \mathrm{k}, \mathrm{k}, \mathrm{l}}$ \\
\hline & {$[10-100]$} & $\begin{array}{l}{[32-} \\
100]\end{array}$ & $\begin{array}{l}{[25-} \\
82]\end{array}$ & $\begin{array}{l}{[15-} \\
55]\end{array}$ & $\begin{array}{l}{[10-} \\
77]\end{array}$ & $\begin{array}{l}{[10-} \\
100]\end{array}$ & \\
\hline \multirow{2}{*}{$\begin{array}{l}\text { Physical component } \\
\text { summary }\end{array}$} & 47 & 52 & 50 & 36 & 30 & 48 & $<0.001^{\mathrm{fj}, \mathrm{j}, \mathrm{k}, \mathrm{l}}$ \\
\hline & {$[16-65]$} & {$[42-65]$} & $\begin{array}{l}{[30-} \\
62]\end{array}$ & $\begin{array}{l}{[23-} \\
43]\end{array}$ & $\begin{array}{l}{[16-} \\
40]\end{array}$ & $\begin{array}{l}{[16-} \\
65]\end{array}$ & \\
\hline \multirow{2}{*}{$\begin{array}{l}\text { Mental component } \\
\text { summary }\end{array}$} & 43 & 46 & 45 & 36 & 40 & 43 & $0.008^{\mathrm{fj,j,k,l}}$ \\
\hline & {$[19-60]$} & [25-59] & $\begin{array}{l}{[19-} \\
59]\end{array}$ & $\begin{array}{l}{[20-} \\
43]\end{array}$ & $\begin{array}{l}{[22-} \\
60]\end{array}$ & $\begin{array}{l}{[19-} \\
60]\end{array}$ & \\
\hline \multirow[t]{2}{*}{ Type 2 score } & 6 & 4 & 4 & 18 & 20 & 6 & $<$ \\
\hline & {$[0-31]$} & {$[0-13]$} & {$[0-14]$} & $\begin{array}{l}{[15-} \\
31]\end{array}$ & $\begin{array}{l}{[15-} \\
31]\end{array}$ & $\begin{array}{l}{[0-} \\
31]\end{array}$ & $0.001^{\mathrm{a}, \mathrm{b}, \mathrm{e}, \mathrm{n}}$ \\
\hline \multicolumn{8}{|c|}{ Values shown are median [IQR], or $\mathrm{n}(\%)$. } \\
\hline \multicolumn{8}{|c|}{ SLE: systemic lupus erythematosus; Hb: haemoglobin. } \\
\hline \multicolumn{8}{|c|}{ 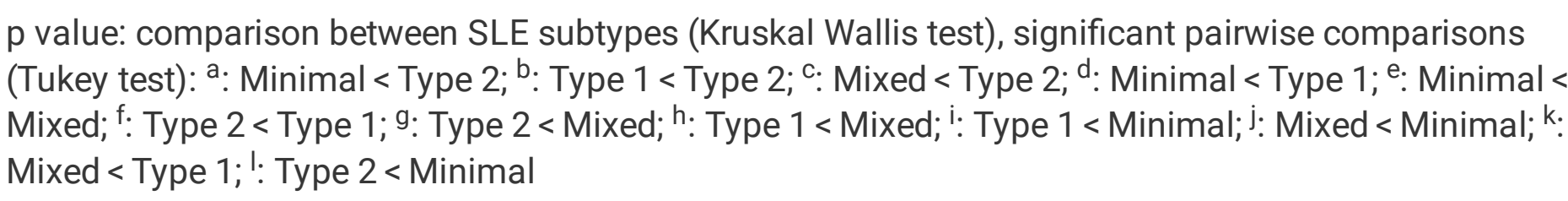 } \\
\hline
\end{tabular}

\section{Clinical groups and immunological activity}

Immunological activity (elevated anti-dsDNA antibodies, anti-dsDNA level, low C3 and/or C4) did not differ between the four clinical groups (Table 3).

\section{Clinical groups and transcriptomic signatures}

Modular IFN score, number of activated IFN modules and level of regulation of each IFN module did not differ between the four clinical groups (Table 4). In the analyses of all modules, the values of two modules (M3.3, cell cycle signature, and M4.14, monocyte signature) were slightly higher in patients in the "mixed" group compared to other groups, but these modules were not significantly activated (Supplementary Tables S1 and S2). 
Table 4

Blood modular IFN signature and SLE clinical groups.

\begin{tabular}{|c|c|c|c|c|c|c|}
\hline & $\begin{array}{l}\text { Minimal } \\
(n=39)\end{array}$ & $\begin{array}{l}\text { Type } 1 \\
(n=37)\end{array}$ & $\begin{array}{l}\text { Type } 2 \\
(n=9)\end{array}$ & $\begin{array}{l}\text { Mixed } \\
(n=15)\end{array}$ & $\begin{array}{l}\text { Total } \\
(n=100)\end{array}$ & $p$ value \\
\hline IFN signature & & & & & & 0.747 \\
\hline Absent & $6(15.4)$ & $6(16.2)$ & $1(11.1)$ & $1(6.7)$ & $14(14)$ & \\
\hline Mild & $3(7.7)$ & 7 (18.9) & 1 (11.1) & 2 (13.3) & $13(13)$ & \\
\hline Moderate & $22(56.4)$ & $14(37.8)$ & $6(66.7)$ & $6(40.0)$ & $48(48)$ & \\
\hline Strong & $8(20.5)$ & $10(27.0)$ & 1 (11.1) & $6(40.0)$ & $25(25)$ & \\
\hline \multicolumn{7}{|l|}{ IFN modules } \\
\hline M1.2 & $33(84.6)$ & 31 (83.8) & 8 (88.9) & 14 (93.3) & $86(86)$ & 0.918 \\
\hline M3.4 & $30(76.9)$ & $24(64.9)$ & 7 (77.8) & $12(80.0)$ & $73(73)$ & 0.611 \\
\hline M5.12 & $8(20.5)$ & $10(27.0)$ & $1(11.1)$ & $6(40.0)$ & $25(25)$ & 0.396 \\
\hline \multicolumn{7}{|l|}{ IFN modules } \\
\hline \multirow[t]{2}{*}{ M1.2 } & 69.4 & 69 & 69.4 & 77.8 & 69.4 & 0.337 \\
\hline & {$[-22.2-92]$} & {$[-11.1-88.9]$} & [-8.3-91.7] & [0-88.9] & {$[-22.2-92]$} & \\
\hline \multirow[t]{2}{*}{ M3.4 } & 38.7 & 31 & 37.1 & 50 & 38.7 & 0.255 \\
\hline & [-6.5-79] & [-1.6-75.8] & [-4.8-71] & [0-69.4] & [-6.5-79] & \\
\hline \multirow[t]{2}{*}{ M5.12 } & 6 & 8 & 5 & 19 & 6 & 0.175 \\
\hline & [-2-65] & {$[-2-76]$} & {$[0-62]$} & {$[0-62]$} & [-2-65] & \\
\hline Values shown & re median [I & R], or n (\%) & & & & \\
\hline
\end{tabular}

\section{Discussion}

This is the first study using items from the SF-36 to assess the ability of a type 2 score to categorize SLE patients according to the presence of symptoms of fatigue, widespread pain and depression. Using the SLEDAI score to define type 1 symptoms, SLE patients from the LUPUCE cohort were categorized into the four groups defined by Duke University, with a similar patient distribution. The differences between these groups were then explored and apart from clinical differences, no significant immunological or transcriptomic profiles associated with the categories were found. 
The initial definition of type 2 symptoms relied on the ACR fibromyalgia criteria (12). Although these criteria are likely to reflect type 2 symptoms accurately, they are rarely available in clinical trials and in historical SLE cohorts. In contrast, SF-36 is widely used to assess HRQoL in SLE (3), as in many chronic diseases (18), and an item-level analysis has been shown to be useful to interpret HRQoL variations in SLE patients (17). We chose to select the most relevant items from the SF-36 questionnaire to define type 2 symptoms and build a score that was easy to use in other cohorts. The values of the type 2 score observed in the LUPUCE cohort were very similar to those from an independent cohort of SLE patients, the PSY-LUP cohort. In addition, the proportions of patients categorized into each of the four clinical groups using the SLEDAI and the type 2 score were almost the same in the LUPUCE cohort and in the cohort described by Duke University. This constitutes an external validation of the SF-36-derived type 2 score and could allow standardization of the clinical categorization of SLE patients into different cohorts. Until now, the clinical segregation of SLE patients was heterogeneous between different studies. Before the first description of type 1/type 2 symptoms by Duke University (10), some studies described SLE patients with active/inactive disease and high/low fatigue (7) and found quite similar proportions as with the type 1/type 2 categorization (19).

Interestingly, as in the cohort from Duke University, no significant difference was observed between the immunological profiles of the four clinical groups. This highlights the fact that categorization into these four clinical groups, instead of providing redundant information, is a complimentary tool for the care of SLE patients and for patient stratification in clinical trials. Patients with type 2 symptoms, regardless of clinical activity, are likely to report higher activity levels than that evaluated by physicians, which can result in discordant measures (10) challenging the interpretation of clinical trial results.

This is the first study to investigate the association between transcriptional signatures, particularly IFN signature, and clinical type 1/type 2 SLE categorization. We could have expected a higher IFN signature in patients with active SLE (type 1 and mixed groups). However, there was no significant difference in IFN signatures between patients with active SLE and patients with inactive SLE. Alternatively, we could also have expected higher IFN signatures in patients reporting fatigue (type 2 group). Indeed, IFN signature was elevated in patients with fibromyalgia $(20,21)$, and therapeutic IFN is associated with neuropsychiatric side-effects such as fatigue and depression (22). In contrast, we have previously shown a paradoxical association between HRQoL and IFN signatures, patients with higher IFN scores displaying better HRQoL in some SF-36 domains (mental health and vitality) (14). However here, although a majority of patients from the type 2 group had a moderate IFN score, no significant difference was observed with the other groups. This is consistent with the report of an absence of correlation between proinflammatory cytokines (including IFN) and HRQoL in SLE patients (23). The same has been observed in patients with primary Sjögren syndrome in whom there was no correlation between IFN signature and HRQoL/fatigue (24). Thus, the IFN signature, as an immunological marker, is not relevant to discriminate type 1 from type 2 profiles.

Our study has some limitations. First, we used items from the SF-36 questionnaire to assess fatigue and depression, and not more specific questionnaires dedicated to SLE patients (such as SLEQOL, LIT, L-QOL, 
LupusQOL, LupusPRO) (25). However, the SF-36 has been used for a long time and more widely than specific scales and it is well validated in SLE (26) and widely available, even retrospectively, in SLE cohorts. Second, the limited number of patients could lead to a loss of power after the division of the cohort into the four clinical groups. New studies with larger populations are therefore needed to confirm our results. Cohorts such as the LUPUCE cohort, providing well-characterized clinical, immunological, transcriptomic and HRQoL data, are very much needed.

\section{Conclusion}

A score derived from the SF-36 can be used to identify SLE patients with type 2 symptoms, and, with the use of the SLEDAl, to categorize patients into four clinical groups. This categorization, which is not related to immunological activity or blood transcriptomic profiles, could be useful in the daily care of patients, as well as in clinical trials, for the upstream stratification of patients and for the interpretation of results.

\section{List Of Abbreviations}

ACR: American College of Rheumatology

HRQoL: health-related quality of life

IFN: interferon

SF-36: 36-item short form survey

SLE: systemic lupus erythematosus

SLEDAl: SLE disease activity index

\section{Declarations}

\section{Ethics approval and consent to participate}

This study involved data from patients included in the LUPUCE study (NCT00920114) and the PSY-LUP study (NCT03913754). All patients gave their written informed consent before any study-related procedure. The study was conducted according to the Declaration of Helsinki. The LUPUCE study was approved in France by the Comité de Protection des Personnes Sud Méditerranée 1 (IDRCB 2009-A0025750 ) and in the USA by the Institutional Review Boards (IRBs) of the Baylor Institute of Immunology Research (IRB 011-173) and the Benaroya Research Institute (IRB 12085). The PSY-LUP study was approved by the Comité de Protection des Personnes lle de France I (IDRCB A02747-48).

\section{Availability of data and materials}


Transcriptomic data from the LUPUCE study are deposited in the NCBI Gene Expression Omnibus (GEO, http://www.ncbi.nlm.nih.gov/geo, GEO Series accession number GSE49454). The analyses performed in the current study are available from the corresponding author on reasonable request.

\section{Competing interests}

The authors declare that they have no competing interests.

\section{Funding}

The LUPUCE study was funded by a research grant from the AP-HM (AORC 2008 2009-04). The Psy-LUP study was funded by a research grant "Sciences humaines et sociales \& maladies rares" from the Fondation Maladies Rares (SHS5-MR - H20171206).

\section{Authors' contributions}

R.A., L.C. and N.J.-C. elaborated the type 2 score. E.J. performed the analyses and made the tables and figure. All authors discussed and interpreted the results. R.A. wrote the first draft of the manuscript. L.C. and N.J.-C. corrected the manuscript. All authors approved the final version of the manuscript and agreed to its publication.

\section{Acknowledgements}

We thank Julien Faraut, Marie-Anastasie Aim and Lionel Dany for their help in the PSYLUP study. We thank all investigators of the LUPUCE study. We thank all patients who participated in the LUPUCE and PSYLUP studies.

\section{References}

1. Tektonidou MG, Lewandowski LB, Hu J, Dasgupta A, Ward MM. Survival in adults and children with systemic lupus erythematosus: a systematic review and Bayesian meta-analysis of studies from 1950 to 2016. Ann Rheum Dis 2017;76:2009-16.

2. Kiani AN, Petri M. Quality-of-life measurements versus disease activity in systemic lupus erythematosus. Curr Rheumatol Rep 2010;12:250-8.

3. Devilliers H, Amoura Z, Besancenot J-F, Bonnotte B, Pasquali J-L, Wahl D, et al. Responsiveness of the 36-item Short Form Health Survey and the Lupus Quality of Life questionnaire in SLE. Rheumatology (Oxford) 2015;54:940-9.

4. Cleanthous S, Tyagi M, Isenberg DA, Newman SP. What do we know about self-reported fatigue in systemic lupus erythematosus? Lupus 2012;21:465-76.

5. Schneider M. Pitfalls in lupus. Autoimmun Rev 2016;15:1089-93.

6. Arnaud L, Gavand PE, Voll R, Schwarting A, Maurier F, Blaison G, et al. Predictors of fatigue and severe fatigue in a large international cohort of patients with systemic lupus erythematosus and a 
systematic review of the literature. Rheumatology (Oxford) 2019;58:987-96.

7. Moazzami M, Strand V, Su J, Touma Z. Dual trajectories of fatigue and disease activity in an inception cohort of adults with systemic lupus erythematosus over 10 years. Lupus 2021:961203320983892.

8. Moon S-J, Kang KY, Kwok S-K, Ju JH, Hong Y-S, Park S-H, et al. Differences in quality of life determinants according to the presence of fibromyalgia in middle-aged female patients with systemic lupus erythematosus: a multicenter, cross-sectional, single-ethnicity cohort. Int J Rheum Dis 2018;21:1173-84.

9. Singh JA, Shah NP, Mudano AS. Belimumab for systemic lupus erythematosus. Cochrane Database Syst Rev 2021;2:CD010668.

10. Pisetsky DS, Clowse MEB, Criscione-Schreiber LG, Rogers JL. A novel system to categorize the symptoms of systemic lupus erythematosus. Arthritis Care Res (Hoboken) 2019;71:735-41.

11. Rogers JL, Eudy AM, Pisetsky D, Criscione-Schreiber LG, Sun K, Doss J, et al. Using clinical characteristics and patient-reported outcome measures to categorize systemic lupus erythematosus subtypes. Arthritis Care Res (Hoboken) 2021;73:386-93.

12. Wolfe F, Clauw DJ, Fitzcharles M-A, Goldenberg DL, Häuser W, Katz RL, et al. 2016 Revisions to the 2010/2011 fibromyalgia diagnostic criteria. Sem Arthritis Rheum 2016;46:319-29.

13. Chiche L, Jourde-Chiche N, Whalen E, Presnell S, Gersuk V, Dang K, et al. Modular transcriptional repertoire analyses of adults with systemic lupus erythematosus reveal distinct type I and type II interferon signatures. Arthritis Rheumatol 2014;66:1583-95.

14. Seguier J, Jouve E, Bobot M, Whalen E, Dussol B, Gentile S, et al. Paradoxical association between blood modular interferon signatures and quality of life in patients with systemic lupus erythematosus. Rheumatology (Oxford) 2020;59:1975-83.

15. Jourde-Chiche N, Whalen E, Gondouin B, Speake C, Gersuk V, Dussol B, et al. Modular transcriptional repertoire analyses identify a blood neutrophil signature as a candidate biomarker for lupus nephritis. Rheumatology (Oxford) 2017;56:477-87.

16. Brazier JE, Harper R, Jones NM, O'Cathain A, Thomas KJ, Usherwood T, et al. Validating the SF-36 health survey questionnaire: new outcome measure for primary care. BMJ 1992;305:160-4.

17. Banchereau R, Hong S, Cantarel B, Baldwin N, Baisch J, Edens M, et al. Personalized immunomonitoring uncovers molecular networks that stratify lupus patients. Cell 2016;165:551-65.

18. Hopman WM, Harrison MB, Coo H, Friedberg E, Buchanan M, VanDenKerkhof EG. Associations between chronic disease, age and physical and mental health status. Chronic Dis Can 2009;29:10816.

19. Arnaud L, Mertz P, Amoura Z, Voll RE, Schwarting A, Maurier F, et al. Patterns of fatigue and association with disease activity and clinical manifestations in systemic lupus erythematosus. Rheumatology (Oxford) 2020 Nov 11;keaa671. doi: 10.1093/rheumatology/keaa671.

20. Ovejero T, Sadones O, Sánchez-Fito T, Almenar-Pérez E, Espejo JA, Martín-Martínez E, et al. Activation of transposable elements in immune cells of fibromyalgia patients. Int J Mol Sci 2020;21. 
21. Dolcino M, Tinazzi E, Puccetti A, Lunardi C. Gene expression profiling in fibromyalgia indicates an autoimmune origin of the disease and opens new avenues for targeted therapy. J Clin Med 2020;9.

22. Marcellin P, Lau GKK, Zeuzem S, Heathcote EJ, Pockros PJ, Reddy KR, et al. Comparing the safety, tolerability and quality of life in patients with chronic hepatitis $B$ vs chronic hepatitis $C$ treated with peginterferon alpha-2a. Liver Int 2008;28:477-85.

23. Raymond WD, Eilertsen GØ, Shanmugakumar S, Nossent JC. The impact of cytokines on the healthrelated quality of life in patients with systemic lupus erythematosus. J Clin Med 2019;8:857.

24. Chiche L, Jourde-Chiche N, Cornec D. Should we finally consider the biopsychosocial model in systemic autoimmune diseases? Arthritis Rheumatol 2021;73:717-8.

25. Izadi Z. Health-related quality of life measures in adult systemic lupus erythematosus. Arthritis Care Res (Hoboken) 2020;72 Suppl 10:577-92.

26. Strand V, Simon LS, Meara AS, Touma Z. Measurement properties of selected patient-reported outcome measures for use in randomised controlled trials in patients with systemic lupus erythematosus: a systematic review. Lupus Sci Med 2020;7:e000373.

\section{Figures}

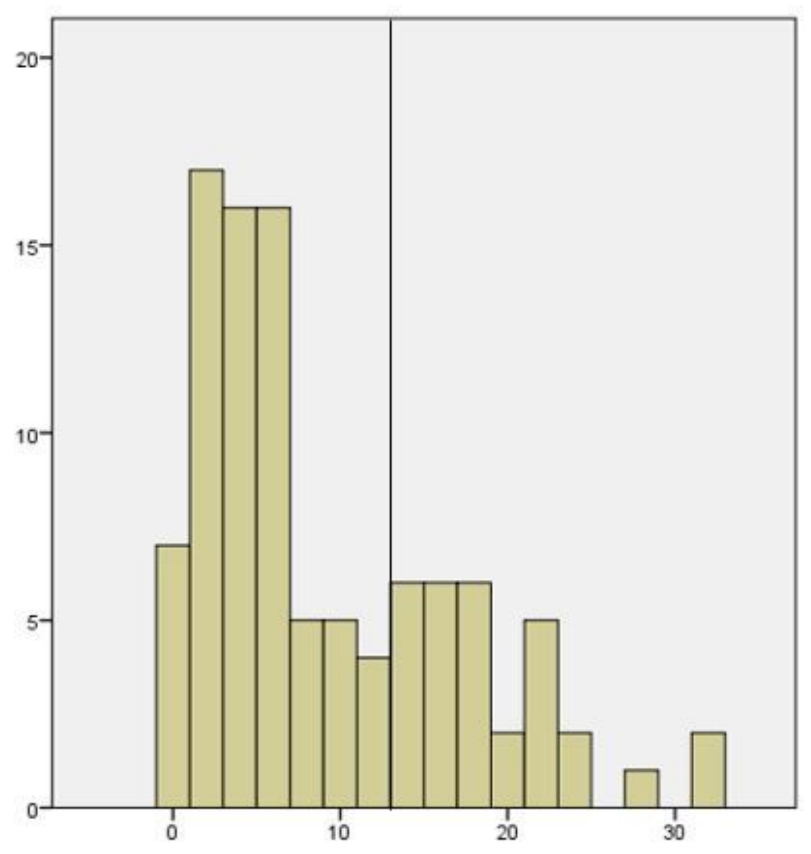

LUPUCE

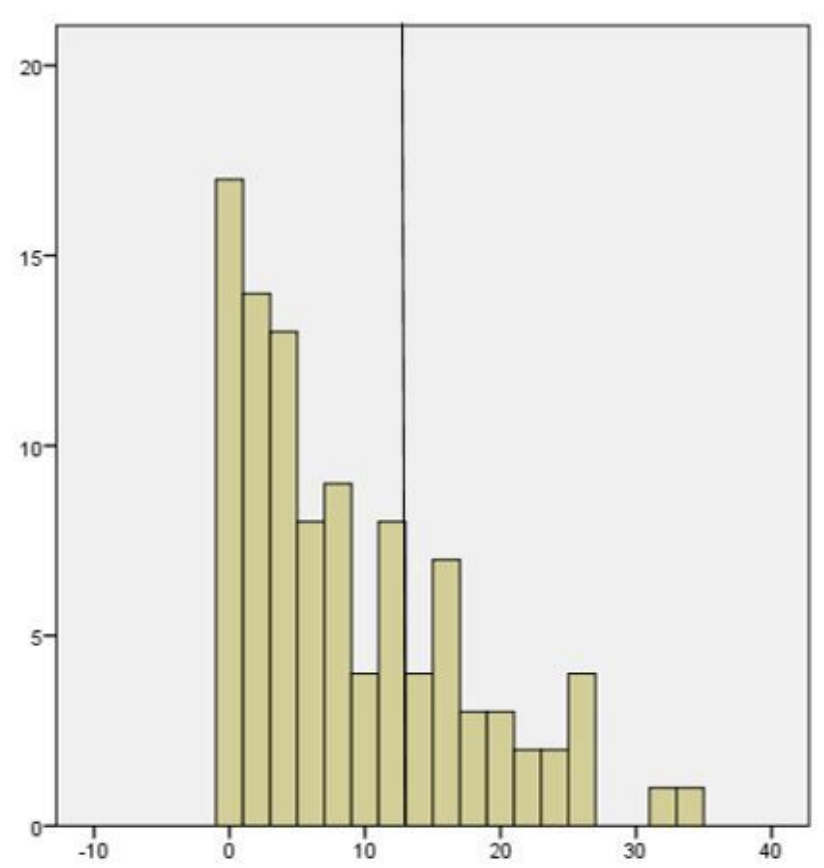

PSY-LUP

Figure 1 
Distribution of type 2 scores in the LUPUCE and PSY-LUP cohorts.

\section{Supplementary Files}

This is a list of supplementary files associated with this preprint. Click to download.

- LUPUCEType1Type2modulesSupplementaryTables.docx 